\title{
Non-Gaussian states from continuous-wave Gaussian light sources
}

\author{
Klaus Mølmer \\ Danish National Research Foundation Center for Quantum Optics \\ Department of Physics and Astronomy \\ University of Aarhus \\ DK 8000 Aarhus C, Denmark \\ (Dated: 11th August 2018)
}

\begin{abstract}
We present a general analysis of the state obtained by subjecting the output from a continuouswave (cw) Gaussian field to non-Gaussian measurements. The generic multimode state of cw Gaussian fields is characterized by an infinite dimensional covariance matrix involving the noise correlations of the source. Our theory extracts the information relevant for detection within specific temporal output modes from these correlation functions. The formalism is applied to schemes for production of non-classical light states from a squeezed beam of light.

PACS numbers: 03.65.Wj; 03.67.-a; 42.50.Dv
\end{abstract}

\section{INTRODUCTION}

Quantum computing and communication, and quantum metrology with atoms and light are topics of high current interest offering on the one hand proposals for new revolutionary technologies and addressing on the other hand fundamental questions about the properties of quantum systems. Light is an ideal carrier of information over long distances, and it is an ideal physical probe for the properties of matter. Numerous proposals have been made for the use of light for quantum information purposes and both theory and experiments have advanced to the quest for and application of specially designed quantum states of light. The observation that coherent states of light suffice for quantum cryptography 1] has not removed the interest in single-photon and other non-classical states, since it has been proven that the entire family of Gaussian states (to which the coherent states belong) cannot be distilled by Gaussian operations [2], and since, e.g., the implementation of qubits for digital quantum computing also necessarily involves non-Gaussian states [3].

Non-Gaussian states can be produced by single photon emitters such as atoms or ions in cavities [4, 5], or $\mathrm{N}-\mathrm{V}$ centers [6] (for a recent review of single-photon emitters see [7]), but they may also result from post selecting the output of Gaussian light sources after non-Gaussian measurements have been carried out on part of the field [8]. Thermal, coherent and squeezed light are all examples of Gaussian states, and prompted by recent experimental and theoretical work on single photon production from a cw squeezed light field [9, 10, 11, 12, 13, 14], we shall in this paper develop a theory for the state of a specific output mode, obtained from a cw Gaussian state light after an arbitrary measurement has been performed on a designated trigger mode. This analysis in fact consists in two straightforward steps:

1. From the general multimode Gaussian state, fully characterized by the correlation functions of the light beam, we derive the covariance matrix and corresponding Gaussian Wigner function for the state of the (trig- ger+output) two mode system prior to detection.

2. The two-mode Wigner function has two complex (four real) arguments, and the effect of the measurement on the trigger mode is described by a (generalized) projection, which amounts to a specific operation on the Wigner function followed by an integration over the trigger mode variables.

These steps, which can both be carried out analytically for several relevant cases, produce a Wigner function for the quantum state of the output mode which provides all available information about the state.

In Sec.II, we show explicity how to extract the twomode Gaussian state from the noise correlations of the Gaussian field light source. In Sec. III, we discuss the state reduction and the output field resulting from different kinds of photon counting measurement on the trigger mode. In Sec. IV, we specialize to the interesting case of single photon production, and we present a concrete analysis for the squeezed output from an OPO and we summarize the technical implementation of various elements and phenomena (beam splitters, losses, inefficient detectors, dark counts, ...). Sec. V concludes the paper.

\section{GAUSSIAN STATES - REDUCTION TO TWO MODES}

In a generic description of the experiments that we wish to analyze, a cw Gaussian light field is split and possibly filtered by a sequence of linear optical devices, and one particular field mode (in our example: the field at a specific instant of time) is registered by a measurement device, see Fig. 1. Conditioned on the measurement output, another component of the beam, and in particular the field pertaining to a single designated output mode, is emitted in a non-Gaussian state. In the case of a low flux from an OPO, the total field contains vacuum and a weakly populated two-photon component, and a single detector click, stemming from the two-photon component, suggests that a one photon state is produced. At higher flux, the squeezed output is a superposition of 


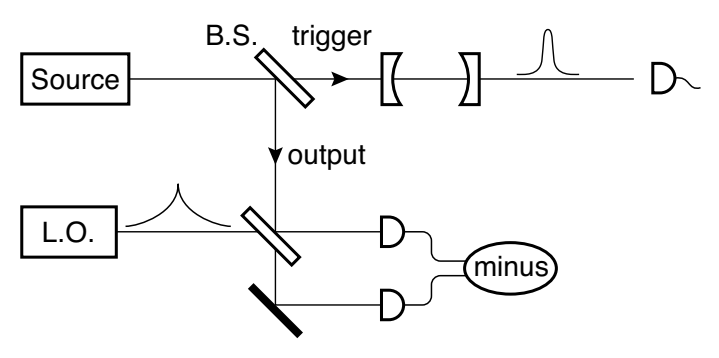

Figure 1: Experimental set-up for production of a nonGaussian state from a Gaussian cw source of light. The field is split into trigger and output components, and after filtering (optional), a non-Gaussian photon-counting detection takes place on the trigger mode. Conditioned on the trigger signal, the output beam may be in a non-classical state, and by use of a temporally modulated local oscillator field, a specific single mode in the output beam can be extracted for homodyne detection.

even photon number states, and a single photon subtraction returns a superposition of only odd number states, which is a non-classical state with some Schrödinger Cat character. This paper addresses how good single photon states and how much non-classical character can be obtained in a realistic physical set-up.

Our analysis proceeds by noting that prior to the count event, we have a system with two relevant field modes:

mode 1: the temporal mode on which the trigger detection takes place. We assume an instant click event at time $t_{c}$, but the field may well be frequency filtered prior to detection, so that the field registered is actually an integral over time of the field that left the original light source.

mode 2: the output mode, populating a quantum state that we shall characterize fully by its phase space Wigner function. The Wigner function is experimentally available by homodyne detection and quantum state tomography [15], where the homodyne detection can be carried out with a time dependent local oscillator field, which will extract the desired output mode function. For our concrete application we expect the optimum mode function to be localized around the time of the click event on mode 1 , and that it has a temporal shape reflecting the band width of the squeezed light source. See Fig.2.

Let $\hat{a}(t)$ and $\hat{a}^{\dagger}(t)$ denote the annihilation and creation operators of the source field. The input to our analysis is the noise correlations represented by the correlation functions $\left\langle\hat{a}(t) \hat{a}\left(t^{\prime}\right)\right\rangle$ and $\left\langle\hat{a}^{\dagger}(t) \hat{a}\left(t^{\prime}\right)\right\rangle$. We do not have to restrict the analysis to this case, but we note that for a stationary source, these correlation functions only depend on the difference between time arguments $(t-$ $\left.t^{\prime}\right)$. The most general formulation of the trigger+output modes and their properties is obtained by designating field mode operators,

$$
\hat{a}_{i}=\int f_{i}\left(t^{\prime}\right) \hat{a}\left(t^{\prime}\right) d t^{\prime}+\xi_{i} \hat{b}_{i, v a c}
$$
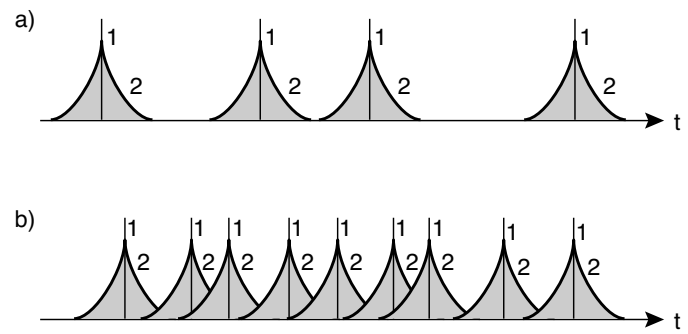

Figure 2: Schematic representation of the trigger (1) and output (2) signals. The OPO produces photons in pairs inside a cavity, and when a discrete trigger photon is detected (vertical lines in the figure), we expect to have a second photon in the output beam, temporally separated from the trigger event according to a mode function (shaded function in the figure) with a width related to the squeezing band width of the source (part a). If the squeezed light source is too bright, the click events occur more often, and the mode functions of the output single photons states start to overlap in an incoherent manner (part b).

where the functions $f_{i}(t)$ with $i=1,2$ denote the mode functions of the trigger and output modes respectively, taking into account completely arbitrary manipulations of the field states by linear filters and beam splitters, and detection in arbitrarily shaped mode functions. The vacuum fields $\hat{b}_{i, v a c}$ are needed for unitarity, but their effects are fully accounted for by the source terms.

With explicit expressions for the functions in (1) and for the source correlations, we can compute all second moments $\left\langle\hat{a}_{i} \hat{a}_{j}\right\rangle,\left\langle\hat{a}_{i}^{\dagger} \hat{a}_{j}\right\rangle$ of the trigger-output modes prior to the trigger detection. The $\mathrm{cw}$ multi-mode field state is Gaussian, and when the state is restricted to only two modes (all others are traced out) the Gaussian property remains. This implies that the two-mode Wigner function of the field modes 1 and 2 is a Gaussian function of four real quadrature variables $\left(y_{1}, y_{2}, y_{3}, y_{4}\right)=$ $\left(x_{1}, p_{1}, x_{2}, p_{2}\right)$, fully characterized by a covariance matrix with elements $\left.\left.V_{i j} \equiv<y_{i} y_{j}\right\rangle+<y_{j} y_{i}\right\rangle$, expressed explicitly in terms of $\left\langle\hat{a}_{i} \hat{a}_{j}\right\rangle,\left\langle\hat{a}_{i}^{\dagger} \hat{a}_{j}\right\rangle$.

$$
W_{V}(\mathbf{y})=\frac{1}{\pi^{2} \sqrt{\operatorname{det}(V)}} \exp \left(-\mathbf{y}^{T} V^{-1} \mathbf{y}\right),
$$

where $\mathbf{y}$ is a column vector, and $\cdot^{T}$ denotes the transpose. (We assume without loss of generality, that there are no mean field components and hence no displacement of the state to take care of).

\section{NON-GAUSSIAN STATES}

The Wigner function (2) is so simple, that it constitutes a natural starting point for analysis of the state of the output mode 2 , conditioned on various possible measurements performed on mode 1 . Homodyne or hetero- 
dyne measurements are readily accounted for by elementary transformations of the covariance matrix, returning a Gaussian state of the output field with a mean amplitude that depends on the trigger measurement outcome, and with a covariance matrix that is independent of the actual outcome 16, 17]. The purpose of the present work is to go beyond the Gaussian states, and for this purpose more general transformations of the Wigner function (2) must be allowed. Such transformations follow if the trigger mode is made subject to a measurement that does not preserve the Gaussian character, for example a photon counting experiment, and we shall analyze three variants of photocounting measurements:

\section{A. Perfect number detector}

A perfect photon number detector will yield an integer eigenvalue, $n=0,1, \ldots$, when applied to the trigger mode. Such a detection event is accompanied by a projection of the field state on the corresponding trigger mode eigenstate with the Wigner function $W_{n}\left(x_{1}, p_{1}\right)$, which for the remaining output mode implies a change into a state described by the conditioned Wigner function

$W_{c, n}\left(x_{2}, p_{2}\right)=\mathcal{N}_{n} \int d x_{1} d p_{1} W_{V}\left(x_{1}, p_{1}, x_{2}, p_{2}\right) W_{n}\left(x_{1}, p_{1}\right)$

where the normalization constants $\mathcal{N}_{n}$ are inversely proportional to the probabilities for the different number state outputs. The most interesting case is probably the one of a detection of a single photon with

$$
W_{n=1}\left(x_{1}, p_{1}\right)=\pi^{-1}\left(2 x_{1}^{2}+2 p_{1}^{2}-1\right) \exp \left(-x_{1}^{2}-p_{1}^{2}\right),
$$

and we observe that the integral (3) only involves a Gaussian and a product of quadratic terms and a Gaussian. It can hence be carried out analytically, the result being itself a product of a Gaussian and a second order poynomial in the variables $x_{2}, p_{2}$. Kim et al [13] have studied the negativity of the Wigner function at the origin as a suitable measure of the non-classicality of the output of such a protocol. Their study dealt only with a physical two-mode system, but they assumed a sufficiently general covariance matrix $V$ that they describe also our general cw setting, where $V$ is the result of an integration over the cw field correlation functions and arbitrary trigger and output mode functions. Their analytical expression for the conditioned Wigner function $W_{c, n=1}\left(x_{2}, p_{2}\right)$ and in particular for its possible negativity at the origin, may be helpful in the search for optimum trigger and output mode functions in such an experiment. Dakna et al [8] have studied the case of a pure single-mode squeezed state, which after beam splitting is a special case of the Gaussian two-mode states, and they have presented various results including output mode Wigner functions conditioned on the detection of different number states of the trigger mode. With Eqs.(23), and the appropriate covariance matrix, determined from the source correlation functions, we are able to generalize these results to search for Schrödinger cat-like states in cw experiments.

\section{B. "On/off" detector}

The so-called on/off detector, is able to distinguish between a vanishing and a non-vanishing photon number in a field mode, but it is unable to resolve photon numbers larger than unity. The interesting "on" output of such a detector, projects the system on the space spanned by all non-vanishing photon number states, equivalent to removal of the vacuum component, and the resulting Wigner function of the output mode is

$$
\begin{array}{r}
W_{c, n}\left(x_{2}, p_{2}\right)=\mathcal{N}_{\text {on }} \int d x_{1} d p_{1} W_{V}\left(x_{1}, p_{1}, x_{2}, p_{2}\right) \\
\left(1-W_{n=0}\left(x_{1}, p_{1}\right)\right),
\end{array}
$$

where $W_{n=0}\left(x_{1}, p_{1}\right)=\pi^{-1} \exp \left(-x_{1}^{2}-p_{1}^{2}\right)$, and the integral is readily evaluated for any covariance matrix $V$. The result is readily seen to be the difference between two Gaussian functions in the variables $x_{2}, p_{2}$. This detector model was recently applied by Sasaki and Suzuki in a theoretical analysis 12 of the cw OPO output field. Their approach did not reduce the system first to the two relevant modes, but used instead a mode expansion on a complete basis of prolate spheroidal wave functions and suitable generating functions for the number distributions studied also by Zhu and Caves [18]. They do, however, also obtain Wigner functions expressed formally as the difference between two Gaussian functions with different pre-factors and widths. Given that both the covariance matrix $V$ and the integral (5) can be evaluated analytically, we advocate that our approach provides a more straightforward analysis of the "on/off" detector.

\section{Click event}

The most natural and realistic detector model, is the one of usual photo detection theory, in which the trigger mode is incident on a physical material, from which a bound electron is excited into the continuum and subsequently detected. In this scenario, it is the electron that is being detected and made subject to a projection into the continuum state space, and the back action on the field state is given by the field annihilation operator [19]. In a density matrix formulation this amount to the transformation $\rho \rightarrow \hat{a}_{1} \rho \hat{a}_{1}^{\dagger} / \operatorname{Tr}\left(\hat{a}_{1}^{\dagger} \hat{a}_{1} \rho\right)$, and using the operator correspondence 20] between application of the operator $\left(\hat{a}_{1}=\left(\hat{x}_{1}+i \hat{p}\right) / \sqrt{2}\right.$ and multiplying and differentiating the Wigner function with respect to its arguments $x_{1}, p_{1}$, we find the non-Gaussian two-mode Wigner function of the field after the click of the detector. The reduced state of the output field is at this point obtained by tracing over the trigger mode field, i.e., by integrating 
the Wigner function with respect to the $x_{1}, p_{1}$ arguments yielding the final expression,

$$
\begin{array}{r}
W_{\text {click }}\left(x_{2}, p_{2}\right)=\mathcal{N}_{\text {click }} \int d x_{1} d p_{1} \\
\frac{1}{2}\left(x_{1}^{2}+p_{1}^{2}+\frac{1}{4}\left(\frac{\partial^{2}}{\partial x_{1}^{2}}+\frac{\partial^{2}}{\partial p_{1}^{2}}\right)+x_{1} \frac{\partial}{\partial x_{1}}+p_{1} \frac{\partial}{\partial p_{1}}+1\right) \\
W_{V}\left(x_{1}, p_{1}, x_{2}, p_{2}\right) .
\end{array}
$$

This is a lengthy expression, but the appearance of terms up to only second order together with the Gaussian function ensures that the result, as in the case of a one-photon detection, is of the form of a Gaussian function multiplied with a second order polynomial with no first order terms in $x_{2}, p_{2}$. In practice, the expressions become unwieldy, but the analytical toolboxes in Mathematica, Matlab, or similar solvers readily deal with the expressions.

\section{RESULTS FOR A REALISTIC EXPERIMENT}

In this section, we consider an optical parametric oscillator (OPO) with mirrors with leakage rates $\gamma_{1}, \gamma_{2}$ and a nonlinear gain $\varepsilon$. The output field from this device has the correlation functions 20]

$$
\begin{aligned}
\left\langle\hat{a}(t) \hat{a}\left(t^{\prime}\right)\right. & =\frac{\gamma_{1}}{\gamma_{1}+\gamma_{2}} \frac{\lambda^{2}-\mu^{2}}{4}\left(\frac{e^{-\mu\left|t-t^{\prime}\right|}}{2 \mu}+\frac{e^{-\lambda\left|t-t^{\prime}\right|}}{2 \lambda}\right) \\
\left\langle\hat{a}^{\dagger}(t) \hat{a}\left(t^{\prime}\right)\right. & =\frac{\gamma_{1}}{\gamma_{1}+\gamma_{2}} \frac{\lambda^{2}-\mu^{2}}{4}\left(\frac{e^{-\mu\left|t-t^{\prime}\right|}}{2 \mu}-\frac{e^{-\lambda\left|t-t^{\prime}\right|}}{2 \lambda}\right)
\end{aligned}
$$

where $\lambda=\frac{1}{2}\left(\gamma_{1}+\gamma_{2}\right)+\varepsilon, \mu=\frac{1}{2}\left(\gamma_{1}+\gamma_{2}\right)-\varepsilon$, and where the time dependent field operators are normalized according to delta-correlated commutators $\left[\hat{a}(t), \hat{a}^{\dagger}\left(t^{\prime}\right)\right]=\delta\left(t-t^{\prime}\right)$.

The trigger mode 1 and output mode 2 are single modes of the field, which are in the most general case written as in Eq. (1) with contributions from the $\mathrm{cw}$ source and from vacuum noise sources. Let us comment on the explicit form of these expressions: In our example we imagine that part of the OPO beam is transmitted with amplitude $\tau$ through a beam splitter and the time dependent annihilation operator for the transmitted field can be written

$$
\hat{a}_{\tau}(t)=\tau \hat{a}(t)+\rho \hat{b}_{v a c}(t)
$$

where $\rho \hat{b}_{v a c}(t)$ is a vacuum noise contribution, needed for unitarity, but causing no complications in the following. Field transmission losses and a finite detector efficiency for the trigger mode can be modelled by the effect of yet another beam splitter that transmits only a certain fraction of the field to a perfect counter and adds extra vacuum contributions, i.e., by a simple reduction of the factor $\tau$ in Eq. (8) and by replacement of $\rho$ by a correspondingly larger number.

Further manipulation of the field prior to detection may be relevant, for example a frequency filter may be installed to avoid contributions from other modes of the OPO, not accounted for by Eq.(7), see for example 21]. A simple frequency filter of width $\gamma$ has an exponential temporal transmission function, and the time dependent annihilation operator for the filtered field can be written

$$
\hat{a}_{\tau}(t)=\tau \gamma \int_{-\infty}^{t} \exp \left(-\gamma\left(t-t^{\prime}\right)\right) \hat{a}\left(t^{\prime}\right) d t^{\prime}+\beta_{1} \hat{b}_{1, v a c}(t)
$$

where $\beta_{1} \hat{b}_{1, v a c}(t)$ is again a short hand for the vacuum noise contribution.

Eq.(9) still represents a cw field, but we now assume that this field is being observed by some photon counting device, and that we can define sharply peaked mode functions centered at different times with corresponding annihilation and creation operators with standard single mode commutators. Three different counting operations were described in the previous section, and in a typical experimental implementation of the click detection the field will be monitored continuously in time, and eventually, after an interval of no clicks, the counting device reports a click at time $t_{c}$. We shall hence focus our attention on the correlations between the particular discrete trigger mode where this happens and the remaining output from the light source. We thus define the single mode operator $\hat{a}_{1}=\int h_{t_{c}}(t) \hat{a}_{\tau}(t) d t$, where $h_{t_{c}}(t)$ is a normalized sharply peaked function around $t=t_{c}$. If $h_{t_{c}}(t)$ is much narrower than the temporal correlations of the source field, its actual shape is insignificant, and in a numerical analysis, one may discretize time in intervals of length $d t$ and simply take $h_{t_{c}}(t)=1 / \sqrt{d t}$ on the time interval including $t_{c}$. We note that $\hat{a}_{1}$ is now fully determined as a specific integral over the source output field annihilation operator and vacuum contributions, defining thus precisely the function $f_{1}\left(t^{\prime}\right)$ in Eq. (1).

The whole purpose of the physical setup is to produce a useful non-Gausssian output state, and the output field, which is the component reflected by the first beam splitter in Fig.1, should now be made subject to investigation, conditioned on the click event on the trigger mode. It should be expected, that our non-classical output state predominantly occupies a temporal mode of the field that left the OPO cavity at the same time as the field component that caused the detector click on the trigger mode, and we will also expect that the mode has a temporal width related to the bandwidth of the OPO cavity as represented by the parameters $\lambda$ and $\mu$ in Eq.(7). Using the correlation functions (7), one may indeed determine the conditioned coherence function $\left\langle\hat{a}^{\dagger}\left(t_{c}\right) \hat{a}^{\dagger}(t) \hat{a}\left(t^{\prime}\right) \hat{a}\left(t_{c}\right)\right\rangle$, which in the spirit of standard photo detection theory describes the coherence properties of the field, conditioned on a click event at time $t_{c}$. This function is readily calculated for the specific correlation functions of the OPO, and for a low flux (small $\varepsilon$ ) one indeed finds that this function factors into a product $u^{*}(t) u\left(t^{\prime}\right)$ with a mode function $u(t)$ falling off symmetrically and exponentially as function of $\left|t-t_{c}\right|$, as illustrated in Fig.2a. For larger fluxes, the function no longer 
factors, pointing to the multi mode character of the field, which we can understand as contributions to the field which are correlated with the not so distant click events at other times, as illustrated in Fig. $2 \mathrm{~b}$.

Our formalism permits the definition of mode functions $u(t)$ of arbitrary shape, and in a homodyne detection setup, the extraction of a the field quadrature distribution of a particular mode is implemented by the corresponding temporal modulation of the local oscillator field. We can model losses and imperfect detectors by introducing the effects of an equivalent beam splitter, and hence the single output field mode 2 is also described by Eq.(1) with an explicit expression given for $f_{2}\left(t^{\prime}\right)$.

With explicit expressions for the functions $f_{i}\left(t^{\prime}\right)$ and the correlations (7), we determine by straightforward integration the correlations between the two discrete modes 1 and 2, and subsequently their covariance matrix $V$. Losses and possible incoherent stray field components which may lead to false counts can also be included effectively in the formalism by a modification of the covariance matrix $V \rightarrow L V L+N$, where a diagonal matrix $L=\operatorname{diag}\left(\sqrt{1-\eta_{1}}, \sqrt{1-\eta_{1}}, \sqrt{1-\eta_{2}}, \sqrt{1-\eta_{2}}\right)$ describes the transmission (or detection) efficiencies $1-\eta_{i}$ for the modes, and where $N=\operatorname{diag}\left(\eta_{1}+\xi_{1}, \eta_{1}+\xi_{1}, \eta_{2}+\xi_{2}, \eta_{2}+\xi_{2}\right)$ describes the corresponding vacuum $\left(\eta_{i}\right)$ and additional $\left(\xi_{i}\right)$ noise contributions, see for example Ref. [17].

We shall now show a few examples of Wigner functions for output fields, obtained for different parameters. In the following we show the result for an OPO with one perfectly reflecting mirror $\gamma_{2}=0$ and we shall provide the coupling strength of the squeezing Hamiltonian $\varepsilon$, and the rates and temporal variation, characterizing the mode functions $f_{i}(t)$, in unit of $\gamma_{1}$.

We take first a weakly excited OPO with $\varepsilon=0.01 \gamma_{1}$. We assume the triggering beamsplitter to have a transmission of $1 \%$, and we frequency filter the trigger field with a width of $\gamma=5 \gamma_{1}$ (has only little effect on the field) before the detection in a narrow $\left(\gamma_{1} d t=0.02\right)$ time window. For simplicity we assume an output mode function of the form $u(t) \propto \exp \left(-\alpha\left|t-t_{c}\right|\right.$, with $\alpha=\gamma_{1} / 2$. No losses are assumed. The Wignerfunction is shown in Fig.3. It looks very much like the Wigner function of a one-photon number state, and we indeed compute the overlap with the one photon state to be as high as $98.82 \%$. In the lower panel of Fig. 3 . we show the results obtained if the output light mode suffers a $25 \%$ loss. The overlap with the one photon state is now reduced to $74.14 \%$. The Wigner function is negative at the origin, with a value of -0.3116 (no losses) and -0.154 (25\% loss), compared to the ideal value $-1 / \pi=-0.3183$.

At higher power levels, the OPO does not produce a one-photon state in coincidence with the click event on the trigger mode, but a higher excited non-classical state. In Fig. 4, we show Wigner functions obtained with the same parameters as in Fig. 3, but with $\varepsilon=0.2 \gamma_{1}$. These figures do not look like one-photon state Wigner functions, but they would be very attractive to produce and study for their non-classical behavior, and in particu-
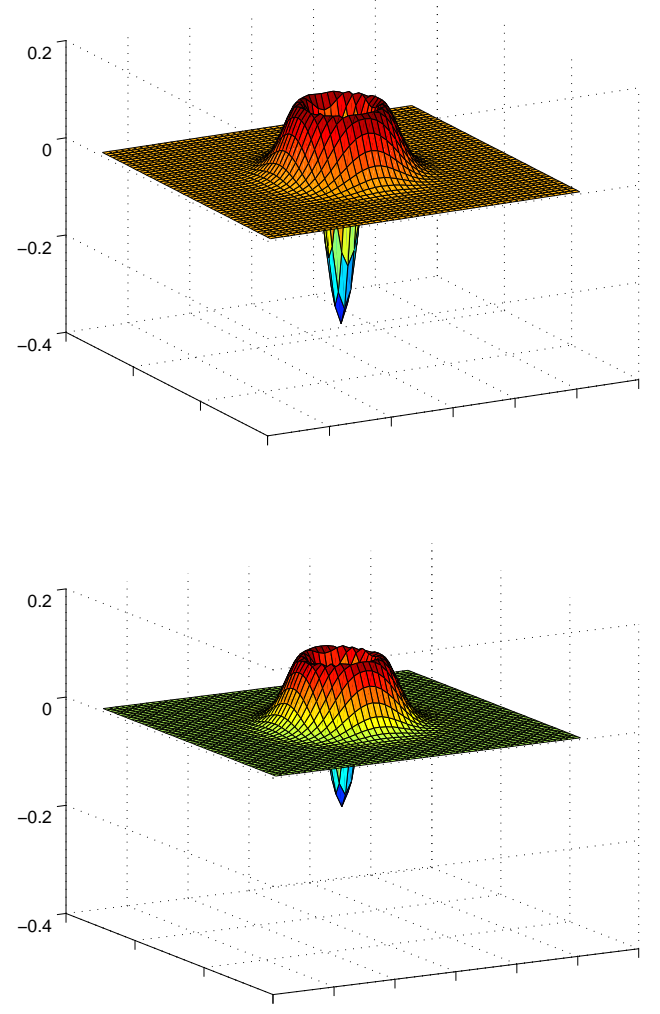

Figure 3: Upper panel: Wigner function for the single mode output field with mode function $\exp \left(-\gamma_{1}\left|t-t_{c}\right| / 2\right)$ conditioned on a detector click in the trigger mode. The field comes from an OPO with $\varepsilon=0.01 \gamma_{1}, \gamma_{2}=0$, and only $1 \%$ of the light is transmitted to the trigger detector. The lower panel shows the Wigner function obtained with the same parameters as in the upper panel but with $25 \%$ loss in the output field.

lar for their double-peaked Schrödinger cat-like character and for the negative values attained at the origin. Without losses, we find the value of the Wigner function at the origin to be -0.2499 in the upper panel and -0.0889 in the lower panel.

We have performed the calculations with the same parameters as in Figs.4, but allowing for a simple variation of the output mode function applied in the analysis. If we choose a modified value of $\alpha=0.337$ instead of 0.5 for the decay rate of the exponential output mode function $\exp \left(-\alpha\left|t-t_{c}\right|\right)$, the negativity at the origin gets slightly deeper $(-0.2618)$ than in the upper panel of Fig.4.There is a host of parameters that can be varied more or less systematically, and if one wishes to produce a specific non-classical state with high fidelity, the present analysis is a good starting point. The natural next step is then to take the mode functions $f_{i}(t)$ as variational functions, and to optimize the quantity of interest (one-photon overlap, negativity at the origin, cat-like behavior, ...) with respect to these functions. 

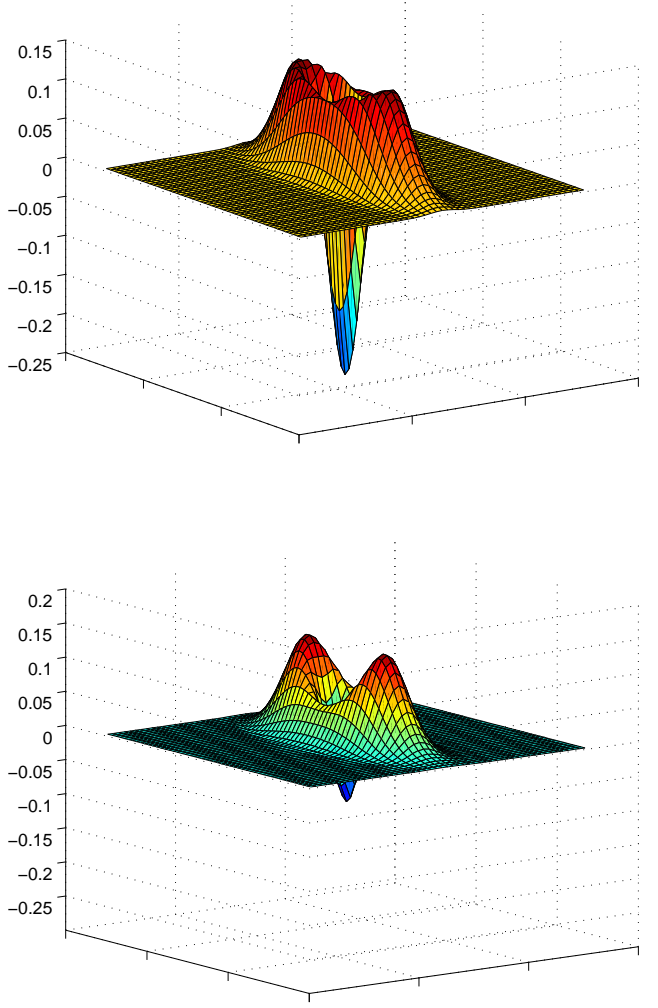

Figure 4: Upper panel: Wigner function for the single mode output field with mode function $\exp \left(-\gamma_{1}\left|t-t_{c}\right| / 2\right)$ conditioned on a detector click in the trigger mode. The field comes from an OPO with $\varepsilon=0.2 \gamma_{1}, \gamma_{2}=0$, and only $1 \%$ of the light is transmitted to the trigger detector. The lower panel shows the Wigner function obtained with the same parameters as in the upper panel but with $25 \%$ loss in the output field.

\section{DISCUSSION}

In conclusion we have established a formalism and we have presented results for the output of cw Gaussian light sources subject to non-Gaussian discrete measurement events on part of the field. The starting point of the analysis is the covariance matrix of the full Gaussian multi-mode field, given explicitly by the continuous time correlation functions (7). The relevant two modes are described by a reduced quantum state, where one should formally trace over all other unobserved modes. The infinite dimensional covariance matrix is then reduced to a covariance matrix for the two modes of interest, and rather than taking the trace, we identify this matrix by calculating explicitly the covariance matrix elements. The Gaussian character of the two-mode state is broken by the measurement, but at this stage, the system is so simple that any realistic trigger mode detection scheme is readily implemented as a formal operation on the two-mode Wigner function of the joint system.
This is illustrated by the action of three different nonGaussian counting operations, which can all be handled analytically due to the simplicity of Gaussian integrals of polynomials. A numerical two-dimensional integral can of course be applied, if need be.

We note that if an experiment is continuously not showing any counting events until time $t_{c}$, these null measurements also have implications on the output state, and one might question the use of the steady state twotime correlation functions in the determination of the output field. In order to rely on steady state correlation functions, one precisely has to run the experiment continuously in time and note that click events will occur after sequences of no-click intervals of varying duration, and that averaging over all click events corresponds to an averaging over all such possible histories, which, in turn, is equivalent to the steady state average of the unprobed system. This justifies the theoretical treatment, but it also suggests, that one may carry out experiments, where the output field is conditioned on a more detailed requirement of the trigger history, for example, that no clicks have occurred for a certain time before $t_{c}$. This scenario will be of relevance if the trigger mode has a high photon detection efficiency (otherwise we automatically ignore many photons and hence de facto work under average steady state conditions). Since the no-click event is a projection on the vacuum state and is hence a Gaussian operation, we may in fact provide a simple theory also for this scenario.

A useful feature of the Gaussian states is the ease at which the number of modes can be increased without rendering the theoretical treatment untractable. This implies that the above analysis readily allows generalization to situations where fields from several Gaussian cw and pulsed sources are included. We imagine in particular, that a recent proposal 14] for production of one-photon states with no pollution from higher number state components involving two relatively intense OPO outputs and a coincidence of two single photon detection events, can be studied under true cw conditions. Such an analysis would imply a second step away from Gaussian states, and it remains to be seen which tools will be optimum for the efficient handling of this progressively more complicated problem.

Despite the fact that the quantum theory of electromagnetic radiation has been well established for a very long time and that all aspects of light generation and detection are quantum optics text book material, a "gap" remains between the description of light as a field and as composed of photons. This gap is not due to the waveparticle duality in quantum theory, but is of a much more pragmatic origin, namely that the expansion of fields in terms of number states is sensible and useful only for a single or few modes, whereas cw fields with infinitely many modes are more conveniently described in terms of the properties of field operators, labelled by continuous mode arguments (typically time or frequency).

The formal equivalence between the Schrödinger pic- 
ture expansion on number states and the Heisenberg picture representation of operators is useful for calculations in the few-mode but not in the cw case. Both the Schrödinger and Heisenberg formalisms can be applied to predict mean values and variances, but the quantum state reduction and dynamics following a detection event at time $t_{c}$, is not well accounted for in the Heisenberg picture, and the dimensionality of the Hilbert space for infinitely many modes makes the Schrödinger description unmanageable. We have previously shown that for analyses restricted to Gaussian states and operations the full multi mode state reduction dynamics is indeed tractable 17, 22, 23]. It is this latter approach that has been extended in the present work, where we explicitly reduce the Gaussian field description of the full multi-mode problem to a Gaussian field description of just two modes, from which the Schrödinger representation of the state reduction mechanism is readily tractable, also in the nonGaussian case.

The author acknowledges discussions with Jonas S. Neergaard-Nielsen, Eugene S. Polzik and Uffe V. Poulsen.
[1] F. Grosshans, G. Van Assche, J. Wenger, R. Brouri, N. J. Cerf, and Ph. Grangier, Nature (London) 421, 238 (2003); Ch. Silberhorn, T. C. Ralph, N. Lütkenhaus, and G. Leuchs, Phys. Rev. Lett. 89, 167901 (2002).

[2] J. Eisert, S. Scheel, and M. B. Plenio, Phys. Rev. Lett. 89, 137903 (2002).

[3] E. Knill, R. Laflamme, and G. J. Milburn, Nature (London) 409, 46 (2001).

[4] A. Kuhn, M. Hennrich, and G. Rempe, Phys. Rev. Lett. 89, 067901 (2002).

[5] M. Keller, B. Lange, K. Hayasaki, W. Lange, and H. Walther, Nature (London) 431, 1075 (2004).

[6] C. Kurtsiefer, S. Mayer, P. Zarda, and H. Weinfurter, Phys. Rev. Lett. 85, 290 (2000); R- Brouri, A. Beveratos, J.-P. Poizat, and P. Grangier, Opt. Lett. 25, 1294 (2000).

[7] M. Oxborrow and A. G. Sinclair, Contemp. Phys. 46, 173 (2005).

[8] M. Dakna, T. Anhut, T. Opatrný, L. Knöll, and D.-G. Welsch, Phys. Rev. A 55, 3184 (1997).

[9] J. Wenger, R. Tualle-Brouri, and P. Grangier, Phys. Rev. Lett. 92, 153601 (2004).

[10] J. S. Neergaard-Nielsen, B. Melholt Nielsen, C. Hettich, K. Mølmer and E. S. Polzik, Generation of a superposition of odd photon number states for quantum information networks, quant-ph/0602198

[11] F. Grosshans and P. Grangier, Eur. Phys. J. D14, 119 (2001).
[12] M. Sasaki and S. Suzuki, Multimode theory of measurement-induced non-Gaussian operation on wideband squeezed light, quant-ph/0512073

[13] M. S. Kim, E. Park, P. L. Knight, and H. Jeong, Phys. Rev. A 71, 043805 (2005).

[14] C. R. Myers, M. Ericsson, and R. Laflamme, A Single Photon Source With Linear Optics and Squeezed States, quant-ph/0408194

[15] U. Leonhardt, Measuring the quantum state of light, Cambridge University Press 1997.

[16] J. Eisert and M. Plenio, Int. J. Quant. Inf. 1, 4792003.

[17] K. Mølmer and L. B. Madsen, Continuous measurements on continuous variable quantum systems: The Gaussian description, in "Quantum Information with Continuous Variables", Eds. G. Leuchs, N. Cerf, and E. Polzik. To be published by Imperial College Press, quant-ph/0511154

[18] C. Zhu and C. M. Caves, Phys. Rev. A 426794 (1990).

[19] R. Glauber, Phys. Rev. 130, 2529 (1963); ibid, 131, 2766 (1963).

[20] C. W. Gardiner and P. Zoller, Quantum Noise, SpringerVerlag Berlin Heidelberg, 2000.

[21] Y. J. Lu and Z. Y. Ou, Phys. Rev. A 62, 033804 (2000).

[22] K. Mølmer and L. B. Madsen, Phys. Rev. A 70, 052102 (2004);

[23] V. Petersen, L. B. Madsen, and K. Mølmer, Phys. Rev. A 72, 053812 (2005). 